\title{
Discussion on Second Language Acquisition in Cognitive Linguistics in the Context of Ecological Discourse
}

\author{
Xin He \\ Nanchang Hangkong University, Nanchang, 330031, China
}

Keywords: Cognitive linguistics, the Context of ecological discourse, Second language acquisition.

\begin{abstract}
Instead of exploring the relationship among language, brain and IQ, cognitive linguistics provides multiple perspectives for common problems in second language acquisition and learning. In this paper, the model of second language acquisition in cognitive linguistics is preliminarily explained in the context of ecological discourse, and an attempt is made to provide accurate and appropriate methods for second language acquisition with cognitive linguistics, providing theoretical hypothesis for further in-depth study of second language acquisition, and finally offering suggestions for establishing a sound theoretical system of second language acquisition.
\end{abstract}

\section{Basic views on second language acquisition in cognitive linguistics}

\subsection{Cognitive linguistics is used to form the basic points of language use and language acquisition through usage}

Take mother tongue as an example. Cognitive language is a kind of complex cognitive activity, which needs to analyze, summarize, conclude and generalize the abstract structure or schema from the concrete things. Second language acquisition is

An acquisition learning, in which students can obtain many symbols from the context of ecological discourse, and then their memory is preserved after analysis and induction. Finally, they will obtain the highest standard of symbols. However, there are two elements to be noted. First, subjective factors include students' learning motivation and attention. Subjective factors refer to students' motivation and attention. Second, objective factors, specific to the language frequency of students, including the form and type frequency. Therefore, in the process of second language acquisition teaching, the specific context should be added, and students' attention should be attracted by means of high-tech and other media. Only by combining the context practice can theory be skillfully applied to daily life.

\subsection{Students' second language acquisition}

The conventional and unconventional structural patterns of students in second language acquisition are similar, which are formed under the condition of general learning mechanism. Researchers believe that students' language development is related to human cognition and social development, including the learning of cultural content, intention recognition and structure mapping, etc. Although the society generally believes that second language acquisition is completed through common learning mechanism, this paper holds a different view: the vast majority of second language learners in society are more or less dependent on classroom learning. Second, the experience is not obtained through actual communication. In addition, more and more scholars is not for studying the language, so the intention recognition and cultural content of the learning effect is small. In classroom teaching, teacher reference materials and some grammar books are provided for students to learn the basic vocabulary and usage, and learners acquire new analogy structure, mapping, and combined structure. Through the subjective guidance of the teacher, by contrast, the effect of second language acquisition is smaller than the native language acquisition's.

The factors influencing second language and first language acquisition are similar, both based on 
the close relationship between frequency cue and result, and the competition generated by multiple cue. Besides, second language acquisition is also easily interfered and hindered by the mother tongue. In the second language classroom, the difference is more obvious. The learner's target language input in classroom learning is obviously insufficient, and the input amount of second language is very small compared with that of first language acquisition, so it is difficult for learners to master the target language, and the second language learning is solidified.

Second language acquisition focuses on language motivation and emphasizes the importance of language motivation in second language acquisition from aspects of iconicity and language awareness. Language motivation is not limited to form and meaning, but also has great application value in many other fields. However, it has not attracted enough attention at present. According to relevant experts, the motivation of language is: the relation between meaning and meaning, and the relation between form and form, and the relation between the meaning and form. The relation between meaning and meaning is quite important, including that the general arrangement is based on prototype image, which can expand the relation degree of prototype image from far to near.

\section{The development of second language acquisition in cognitive linguistics in the context of ecological discourse}

\subsection{Polysemy}

In the process of cognitive language, we find that the meanings of polysemous words do not exist independently, but based on the semantic network system formed by intention as the core. The acquisition of second language tries to explain whether learners' recognition of various relations can help them grasp the meaning of words.

Second language acquisition has something in common with native language acquisition: on the one hand, prototype meaning is easier to acquire than marginal meaning. On the other hand, due to the continuous improvement of students' learning ability, students have a higher accuracy and clarity in choosing the original form of second language. However, even with a higher learning ability, there is still a relatively high gap between students' second language acquisition and their native language acquisition.

Experts conducted three experiments before, in which students majoring in business administration and economics at French universities were studied in order to expand their vocabulary. And these students have similar levels on English. The researchers asked one group to focus on the source domain of the verbs in the material and another group to focus on the verbs themselves. Then

Students were asked to describe the changes of economic situation according to the image, and the number of verb types used by students were calculated. It can be seen that the students in the first group were more abundant in terms of vocabulary than those in the second group, so we know that it is more helpful for students to understand the basic meaning of verbs, which is more conducive to memory retention. But after a period of time, the two groups were tested again and found that the students verb using were almost the same, which showed that this method could not preserve the memory for a long time.

Compared with non-core terms, core terms are more helpful for students to accurately grasp some unfamiliar polysemous words. This is more obvious in long-term memory. In our opinion, core meanings can help students to provide detailed learning process, and some metaphorical meanings can be more appropriately used in the semantic environment of polysemous words, which will be helpful for students to remember in the later periods.

\subsection{Preposition}

Preposition is a typical polysemy. It is very important in cognitive linguistics. Although the preposition is short, it has a wide range of USES, which makes it more difficult for second language learners to learn. Some researchers have found that students who understand the spatial image of prepositions can better interpret the figurative image of the word, and the learning effect is obviously 
better than the use of dictionaries. Some scholars pay attention to the spatial image of prepositions, and combine in-depth cognitive linguistics and the role of lexical knowledge in the second language acquisition process, and take into account the influence of input and migration factors, as well as the role of the input frequency and structural similarity of students in the use of English prepositions at the same time. As they learned through the experiment, the similarity and frequency factors have a bigger influence on the students with low and medium learning ability. For some students with higher learning ability. The influence is relatively small. The words that has the low frequency to use prepositions will not affect the students' second language acquisition either.

Jia Li, Jinting Cai take the principle polysemy network as the prototype. The researchers analyzed the students study for four English prepositions of space, and the researchers surveyed 90 students, and divided them into three groups on average. Sophomore in English major are taken as a secondary learning ability of students, and the postgraduate students are taken as the students of high level, and undergraduates in a British university are taken as the native language learners. Through the pilot study, free language tests, blanks filled with prepositions, talk among the 90 students, it is found that the former two groups of students' perception of prepositional meaning items has not much difference compared to the native language learners. For the prepositional polysemy network learning, they are not very good compared with the native language learners situation. However, the master of core meaning items is much better. In the process of prepositions using, due to the different concept of English and Chinese, the obvious learning transfer has occurred.

\subsection{Phrasal verbs}

Being able to grasp verbs effectively is very important for second language acquisition, and is also one of the most urgent problems to be solved at present. The researchers take cognitive linguistics theory as a guiding method to explore teaching, thus improving the acquisition effect of English verbs. Some experts have focused on verb phrases including up and down. they selected 30 Hungarian intermediate English learners. The students in the first group were told about 20 verbs by using different learning methods. The second group was given ten verbs, and was asked to explaine the verbs in detail in cognitive linguistics, so that the students could understand the hidden meaning behind the verbs. Of two groups of students to learn the verb phrases are the same. During the test of these verbs, the achievement of students in first group is nearly 9\%higher than that in the second group. In addition, 10 testers had not learned the phrasal verbs with up and down. However, the achievement in the first group is nearly $25 \%$ higher than that in the second group, thus knowledge can be transferred to the new environment.

Some experts have used many conceptual metaphors in their experiments. The first and second group use different methods to study the verb phrases. The first group students classified according to the implied meaning of verbs, and proposed the concept of synonyms. The second group students put the phrasal verbs in alphabetical order, and learned from authoritative grammar information for reference, then filled in the blanks with verbs. We tested the verbs that students have learned. The results showed that the learning ability of the first group was significantly higher than that of the second group, but there was no significant difference between the two groups in terms of the unlearned phrases. It can be seen that the students in the first group cannot apply the concepts learned to new content learning. To sum up, it is very effective for students to learn by using the implied meaning of verbs for short-term memory. However, at present, specific reasons for this phenomenon have not been proposed. Some researchers think it may be a metaphorical thinking that can arrange and organize verbs, which is more conducive to learning the relatively random words, while others believe that conceptual metaphor is very important for students' vocabulary organization.

\subsection{Term}

Terms are a collection of words, which do not belong to any sensory system, and have strong logicality information and use. According to the research, it is helpful for students to master the concepts behind the terms. It is showed that in the Boers study, 100 Dutch native students studied 18 terms, The experimental group students classified terms in accordance with the underlying concepts. 
Students were given ten minutes for self study, and 15 minutes for group discussion. Later they should fill in the blanks in words. it was found that the achievement of students in experimental group is better than that of the control group, but at the same time, we found that the similar concepts in mother tongue and target language can have the effect of learning positive transfer. At the same time, it is also very easy to cause the students make some mistakes in the process of using the data.

Fuyin Li has proved that the hidden concept expression, the terms methods, the method of conceptual metaphors and image graphic method in the systematic study of English. Through the discussion on these theories, this paper holds that the image schema and conceptual metaphor are very useful for the systematic study and memory. Through five groups' experiments to study the reliability of this method, the experiment process includes preschool tests for the students, the classroom and after-school test and questionnaire survey. A total of 400 freshmen and sophomores in two universities are taken as the research objects. It is showed that the conceptual metaphors and imagery diagrams are very effective for students to learn and memorize systematically. In this study, we adopted a large number of samples, so the results have a certain degree of reliability.

In addition, some people find that the differences of similar source areas in different cultures also have an impact on students' understanding of relevant terms. In the study, we tested 80 middle-level English students from French universities and asked them to guess the meaning of some of the terms. We studied 24 terms covering four areas, which were less transparent and had no corresponding meaning in French. The students were asked to guess the meaning of the terms in 20 minutes without help. It was found that there would be obvious differences in terms of different source domains, so we can conclude that it is more difficult for students to learn when the prominence of source domain in the target language is higher than that in the native language.

\section{Conclusion}

To sum up. From the perspective of cognitive language, the study of the second language system has been formed on a large scale. The complementarity and communication of these two fields provide a large number of available resources and diversified research perspectives for scholars. As the study of second language acquisition in cognitive language environment restricts the support and test of people's thinking and behavior patterns and other abundant data, the application scope of relevant research methods is also expanding, which is very promising in the future.

\section{Acknowledgement}

This work was supported by the 2018 Annual Planning Project of the "Thirteenth Five-Year" of social science of Jiangxi: Multilingual Translation and International Communication of Ecological Discourse and Ecological Culture in China. [Project No.: 18YY14].

\section{References}

[1] Cuihong Yu, Jianfu Liu. New progress in the research paradigm of second language acquisition from the perspective of cognitive linguistics. Modern foreign language, 2015(6):833-841.

[2] Hongxia Jia, Mingjie Huo, Difei Li. New trends in the research on cognitive linguistics and second language acquisition in China -- summary of the second national symposium on cognitive linguistics and second language acquisition. Foreign languages (journal of Shanghai international studies university), 2011, 34(2):95-96. 